\title{
Symboles des restes quadratiques des discriminants dans les extensions modérément ramifiées
}

\author{
par
}

\section{A. Movahhedi et M. Zahidi (Limoges)}

1. Introduction. Soit $L$ un corps de nombres de degré $n$ sur le corps $\mathbb{Q}$ des nombres rationnels de discriminant $D=D_{L / \mathbb{Q}}$. Si l'entier $D$ n'est pas un carré, on note $d$ le discriminant du corps quadratique $\mathbb{Q}(\sqrt{D})$, sinon on pose $d=1$. Soit $p$ un nombre premier non-ramifié dans $L$ de sorte que le symbole des restes quadratiques $\left(\frac{D}{p}\right)$ soit non-nul. Un théorème déjà ancien dû à A. Pellet ([3, page 245]), L. Stickelberger et G. Voronoï montre que la parité du nombre $g$ d'idéaux premiers de $L$ au-dessus de $p$ est déterminée par ce symbole $\left(\frac{D}{p}\right)$. En effet, nous avons $\left(\frac{D}{p}\right)=(-1)^{n-g}$.

Plus généralement, même si $p$ est ramifié dans $L$, on aimerait pouvoir relier le symbole $\left(\frac{d}{p}\right)$ à la décomposition $(p)=\mathfrak{P}_{1}^{e_{1}} \ldots \mathfrak{P}_{g}^{e_{g}}$ de $p$ en produit d'idéaux premiers $\mathfrak{P}_{i}$ de $L$.

Supposons que $p$ n'est pas sauvagement ramifié dans $L$. Si $f_{i}$ désigne le degré résiduel de $\mathfrak{P}_{i}$ dans l'extension $L / \mathbb{Q}$, alors la valuation $p$-adique du discriminant $D$ est donnée par $v_{p}(D)=\sum_{i=1}^{g}\left(e_{i}-1\right) f_{i}$ [9, Chap. 3 , Prop. 13]. Donc le symbole $\left(\frac{d}{p}\right)$ est non-nul dès que tous les indices de ramification $e_{i}$ sont impairs. Dans ce dernier cas, généralisant une série de résultats (Wahlin [10], Hasse [5], Buhler [2], Dribin [4], Kientega [6], ...), $\mathrm{P}$. Barrucand et F. Laubie ont établi la formule suivante (également valable dans le cas relatif) [1] :

$$
\left(\frac{d}{p}\right)=(-1)^{F}\left(\frac{p}{E}\right) \quad \text { avec } \quad E=\prod_{2 \nmid f_{i}} e_{i} \text { et } F=\sum_{2 \mid f_{i}} 1 .
$$

Notre but est de donner une formule analogue sans aucune hypothèse sur la parité des indices de ramification $e_{i}$. Cet article s'inscrit donc comme une suite logique de [1] et en est largement inspiré.

2. Énoncés des résultats. Soient $K$ un corps de nombres et $L$ une extension finie de $K$ de degré $n$. Soit $\left\{b_{1}, \ldots, b_{n}\right\}$ une base du $K$-espace

2000 Mathematics Subject Classification: 11A15, 11R29, 11S15. 
vectoriel $L$. Le discriminant $D=D_{L / K}=\operatorname{det}\left(\operatorname{Tr}_{L / K}\left(b_{i} b_{j}\right)\right)$ est un élément non-nul de $K: D \in K$. La classe $\delta=\delta_{L / K}$ de $D$ modulo les carrés $K^{\cdot 2}$ est indépendante du choix de la base, c'est donc un invariant de l'extension $L / K$; elle détermine une extension quadratique (ou triviale) $K(\sqrt{\delta})$.

Soit $\mathfrak{p}$ un idéal premier de $K$. Notons $K_{\mathfrak{p}}$ le complété de $K$ en la place $\mathfrak{p}$. On va s'intéresser au symbole des restes quadratique $\left(\frac{\delta}{\mathfrak{p}}\right)$ : étant donné $a \in K_{\mathfrak{p}}$, le symbole des restes quadratiques $\left(\frac{a}{\mathfrak{p}}\right)$ est défini par

$$
\left(\begin{array}{l}
a \\
\mathfrak{p}
\end{array}\right)= \begin{cases}1 & \text { si } K_{\mathfrak{p}}(\sqrt{a})=K_{\mathfrak{p}}, \\
-1 & \text { si } K_{\mathfrak{p}}(\sqrt{a}) / K_{\mathfrak{p}} \text { est une extension quadratique non-ramifiée, } \\
0 & \text { si } K_{\mathfrak{p}}(\sqrt{a}) / K_{\mathfrak{p}} \text { est une extension quadratique ramifiée. }\end{cases}
$$

En particulier $\left(\frac{a}{\mathfrak{p}}\right)$ ne dépend que de la classe de $a$ modulo les carrés. Soit $\mathfrak{p}=\mathfrak{P}_{1}^{e_{1}} \ldots \mathfrak{P}_{g}^{e_{g}}$ la décomposition de l'idéal $\mathfrak{p}$ en produit d'idéaux premiers deux à deux distincts $\mathfrak{P}_{i}$ de $L$. On note $f_{i}$ le degré résiduel de $\mathfrak{P}_{i}$ de sorte que $n=e_{1} f_{1}+\ldots+e_{g} f_{g}$. On désigne par $\pi \in \mathfrak{p}$ une uniformisante du corps local $K_{\mathfrak{p}}$.

Proposition 2.1. On suppose que l'idéal premier $\mathfrak{p}$ de $K$ est non 2adique. Si $\sum_{2 \mid e_{i}} f_{i}$ est un entier pair, alors le produit $\prod_{2 \mid e_{i}}\left(\frac{\pi}{\mathfrak{P}_{i}}\right)$ est non-nul et est indépendant du choix de l'uniformisante $\pi$.

Cette proposition suggère

DÉfinition 2.2. Pour tout idéal premier non 2-adique $\mathfrak{p}$ du corps de nombres $K$, on pose

$$
\varepsilon(\mathfrak{p})=\varepsilon_{L / K}(\mathfrak{p})= \begin{cases}0 & \text { si } \sum_{2 \mid e_{i}} f_{i} \text { est impair } \\ \prod_{2 \mid e_{i}}\left(\frac{\pi}{\mathfrak{P}_{i}}\right) & \text { sinon, }\end{cases}
$$

où $\pi$ désigne une uniformisante quelconque du corps local $K_{\mathfrak{p}}$. Si tous les $e_{i}$ sont impairs, on convient que $\varepsilon(\mathfrak{p})=1$. En particulier $\varepsilon(\mathfrak{p})=1$ dès que l'idéal premier $\mathfrak{p}$ est non-ramifié dans $L$.

Remarque 2.3. Le symbole $\varepsilon$ peut être interprété par l'application de réciprocité d'Artin de la manière suivante. Notons $\mathfrak{A}$ l'idéal $\prod_{2 \mid e_{i}} \mathfrak{P}_{i}$ de $L$. Soit $(\mathfrak{A}, L(\sqrt{\pi}) / L)$ l'élément du groupe de Galois $G(L(\sqrt{\pi}) / L)$ défini par le symbole d'Artin. Lorsque $\varepsilon_{L / K}(\mathfrak{p})$ est non-nul, il est égal à 1 si et seulement si le symbole d'Artin $(\mathfrak{A}, L(\sqrt{\pi}) / L)$ est l'identité [8, Chap. IV, $\S 8]$. Nous utiliserons fréquemment cette caractérisation de $\varepsilon_{L / K}(\mathfrak{p})$.

A l'aide des propriétés fonctorielles du symbole d'Artin, nous pouvons établir une formule de transitivité pour $\varepsilon$ : 
Proposition 2.4. Soit $K \subset M \subset L$ une tour d'extensions de corps de nombres. Soit $\mathfrak{p}$ un idéal premier de $K$. Supposons que $\varepsilon_{L / K}(\mathfrak{p}), \varepsilon_{M / K}(\mathfrak{p})$ ainsi que les $\varepsilon_{L / M}(\mathcal{P})$ pour $\mathcal{P} \mid \mathfrak{p}$ sont non-nuls. Alors nous avons

$$
\varepsilon_{L / K}(\mathfrak{p})=\varepsilon_{M / K}(\mathfrak{p})^{[L: M]} \prod_{\substack{\mathcal{P} \mid \mathfrak{p} \\ 2 \nmid e(\mathcal{P} / \mathfrak{p})}} \varepsilon_{L / M}(\mathcal{P}) .
$$

Le théorème suivant est le résultat principal de cet article qui relie les deux symboles $\left(\frac{\delta_{L / K}}{\mathfrak{p}}\right)$ et $\varepsilon_{L / K}(\mathfrak{p})$.

ThÉORÈme 2.5. Soit $\mathfrak{p}$ un idéal premier non 2-adique du corps de nombres $K$. On suppose que $\mathfrak{p}$ n'est pas sauvagement ramifié dans L. Alors les symboles $\left(\frac{\delta_{L / K}}{\mathfrak{p}}\right)$ et $\varepsilon_{L / K}(\mathfrak{p})$ sont reliés par la formule

$$
\left(\frac{\delta_{L / K}}{\mathfrak{p}}\right)=(-1)^{F+(q-1) G / 2}\left(\frac{q}{E}\right) \varepsilon_{L / K}(\mathfrak{p})
$$

où $q$ est la norme absolue de $\mathfrak{p}$ et les trois entiers $E, F$ et $G$ sont définis par

$$
E=\prod_{2 \nmid e_{i} f_{i}} e_{i}, \quad F=\sum_{\substack{2 \mid f_{i} \\ 2 \nmid e_{i}}} 1, \quad G=\sum_{\substack{4 \mid e_{i} \\ 2 \nmid f_{i}}} 1 .
$$

La démonstration de ce théorème se fait essentiellement en trois étapes : complétion, dévissage et globalisation.

Remarque 2.6. (i) Lorsque tous les indices de ramification $e_{i}$ sont impairs, alors $G=0, \varepsilon_{L / K}(\mathfrak{p})=1$ et on retrouve le théorème principal de Barrucand-Laubie [1, Théorème 2].

(ii) Pour les idéaux premiers 2-adiques $\mathfrak{p}$ qui ne sont pas sauvagement ramifiés dans $L$, nous avons encore

$$
\left(\frac{\delta_{L / K}}{\mathfrak{p}}\right)=(-1)^{F}\left(\frac{q}{E}\right) .
$$

Néanmoins, ils ont été exclu de l'énoncé du théorème précédent car pour ces idéaux $\varepsilon$ n'est pas défini.

Supposons maintenant que l'extension $L / K$ est galoisienne de groupe de Galois $G$. Soit, comme d'habitude,

$e=$ l'indice de ramification de $\mathfrak{p}$ dans $L / K$,

$f=$ le degré résiduel de $\mathfrak{p}$ dans $L / K$,

$g=$ le nombre d'idéaux premiers de $L$ au-dessus de $\mathfrak{p}$.

Alors pour chaque uniformisante $\pi \in \mathfrak{p}-\mathfrak{p}^{2}$, le symbole $\varrho:=\left(\frac{\pi}{\mathfrak{P}}\right)$ est indépendant du choix de la place $\mathfrak{P}$ au-dessus de $\mathfrak{p}$ de sorte que $\varepsilon_{L / K}(\mathfrak{p})=\varrho^{g}$ est une puissance $g$-ième. 
Avec les notations ci-dessus, le théorème 2.5 montre facilement que la valeur du symbole $\left(\frac{\delta_{L / K}}{\mathfrak{p}}\right)$ est donnée par

Corollaire 2.7. Supposons que L est une extension galoisienne de $K$. Pour tout idéal premier $\mathfrak{p}$ de $K$ qui n'est pas sauvagement ramifié dans $L$, nous avons

$$
\left(\frac{\delta_{L / K}}{\mathfrak{p}}\right)= \begin{cases}0 & \text { si } 2 \mid \text { e et } 2 \nmid f g \\ \varrho^{g} & \text { si } 2 \mid \text { e et } 2 \mid f g \\ (-1)^{g} & \text { si } 2 \nmid \text { e et } 2 \mid f g \\ \left(\frac{q}{e}\right) & \text { si } 2 \nmid n\end{cases}
$$

Dans la situation de ce dernier corollaire, le cas où $2|e, 2| f$ et $2 \nmid g$ est le seul où la connaissance des entiers $e, f$ et $g$ ne suffit pas pour déterminer la valeur de $\left(\frac{\delta_{L / K}}{\mathfrak{p}}\right)$. Dans ce dernier cas, nous avons $\left(\frac{\delta_{L / K}}{\mathfrak{p}}\right)=\varepsilon_{L / K}(\mathfrak{p})=$ $\left(\frac{\pi}{\mathfrak{P}}\right) \neq 0$. La valeur de $\varrho=\left(\frac{\pi}{\mathfrak{P}}\right)$ est alors liée à la structure du groupe de décomposition $D=D(\mathfrak{P} / \mathfrak{p})$ de la place $\mathfrak{P}$ dans l'extension $L / K$. Plus précisément, nous avons

Proposition 2.8. Soit $L / K$ une extension galoisienne de corps de nombres. Soient $\mathfrak{p}$ un idéal premier non 2 -adique de $K$ et $\mathfrak{P}$ un idéal premier de $L$ au-dessus de $\mathfrak{p}$. Supposons que le degré résiduel $f$ de $\mathfrak{p}$ dans $L / K$ est pair. Soit $\pi$ une uniformisante de $K_{\mathfrak{p}}$. Alors $\left(\frac{\pi}{\mathfrak{P}}\right)=1$ si et seulement si le 2-sous-groupe de Sylow du groupe de décomposition $D(\mathfrak{P} / \mathfrak{p})$ n'est pas cyclique.

Notons que dans la même extension $L / K$, il est possible que $\varepsilon$ prenne les trois valeurs $-1,0$ et 1 en trois places ramifiées : Prenons, par exemple, $K=$ $\mathbb{Q}$ et soit $L:=\mathbb{Q}(\sqrt{210+21 \sqrt{10}})$. Alors $L / \mathbb{Q}$ est une extension cyclique de degré 4 où à part 2 , se ramifient uniquement les nombres premiers 3,5 et 7 . Plus précisément, le discriminant de $L$ est donné par $D=2^{11} \cdot 3^{2} \cdot 5^{3} \cdot 7^{2}$. Puisque 3 est décomposé dans $\mathbb{Q}(\sqrt{10})$, il se décompose dans $L$ sous la forme $3=\mathfrak{p}_{1}^{2} \mathfrak{p}_{2}^{2}$, donc d'après le corollaire 2.7 on a $\varepsilon_{L / \mathbb{Q}}(3)=1$. Vu la valuation 5 -adique du discriminant, 5 se ramifie totalement dans $L$, donc toujours d'après le corollaire 2.7 on a $\varepsilon_{L / \mathbb{Q}}(5)=0$. Quant au premier 7 , puisqu'il est inerte dans $\mathbb{Q}(\sqrt{10})$, on a dans $L: 7=\mathfrak{p}^{2}$, donc $\varepsilon_{L / \mathbb{Q}}(7)=-1$ grâce au corollaire 2.7 et la proposition 2.8 .

Comme conséquence immédiate du corollaire 2.7, citons la proposition suivante qui est à rapprocher au théorème de Pellet-Stickelberger-Voronoï.

Proposition 2.9. Soit $K$ un corps de nombres, $L$ une extension galoisienne de $K$ et $\mathfrak{p}$ un idéal premier de $K$ qui n'est pas sauvagement ramifié dans $L$. Si $\left(\frac{\delta_{L / K}}{\mathfrak{p}}\right) \neq 1$, alors le nombre d'idéaux premiers de $L$ au-dessus de $\mathfrak{p}$ est impair. 
Il n'est pas difficile de voir que la réciproque de la proposition précédente est inexacte : en effet, il suffit de prendre $K=\mathbb{Q}, L=\mathbb{Q}(\sqrt{2}, \sqrt{3})$ et $\mathfrak{p}=3 \mathbb{Z}$. Alors $\mathfrak{p}=\mathfrak{P}^{2}$ dans $L$ et nous avons $\left(\frac{\delta_{L / K}}{\mathfrak{p}}\right)=1$.

3. Étude locale. Dans cette section, nous ne considérons que des corps locaux, c'est-à-dire complets pour une valuation discrète et ayant un corps résiduel fini. Étant donné un corps local $E$, nous notons

$\pi_{E}=\pi=$ une uniformisante de $E$;

$A_{E}=$ l'anneau de valuation de $E$;

$\mathfrak{p}_{E}=\pi A_{E}$ l'idéal de valuation de $E$;

$q=q_{E}=$ le cardinal du corps résiduel $A_{E} / \mathfrak{p}_{E}$.

Lorsque $F$ est une extension finie séparable du corps local $E$, on désignera comme dans le cas global $\delta_{F / E}$ la classe modulo les carrés du discriminant d'une $E$-base de $F$.

Supposons que $F / E$ est une extension modérément ramifiée d'indice de ramification $e$. Si l'on suppose que $e$ est pair, alors l'extension $F(\sqrt{\pi}) / F$ est non-ramifiée de sorte que $\left(\frac{\pi}{\mathfrak{p}_{F}}\right) \neq 0$ bien que $\left(\frac{\pi}{\mathfrak{p}_{E}}\right)=0$.

LeMme 3.1. On suppose que l'extension locale F/E est totalement et modérément ramifiée de degré pair e. Alors pour toute uniformisante $\pi$ de $E$, il existe une unité $u_{\pi}$ de $E$ telle que

(i) $\delta_{F / E}=u_{\pi} \pi \bmod E^{\cdot 2}$;

(ii) $\left(\frac{u_{\pi}}{\mathfrak{p}_{E}}\right)=\left(\frac{-1}{q_{E}}\right)^{e / 2+1}\left(\frac{\pi}{\mathfrak{p}_{F}}\right)$.

Démonstration. La valuation $\mathfrak{p}_{E}$-adique de l'idéal discriminant de l'extension $F / E$ est égale à $(e-1)$. Comme $e$ est supposé pair, on en déduit qu'il existe une unité $u_{\pi}$ de $E$ telle que $\delta_{F / E}=u_{\pi} \pi \bmod E^{\cdot 2}$.

L'extension $F / E$ étant modérée, il existe une uniformisante $\pi^{\prime}$ de $E$ telle que $F=E\left(\sqrt[e]{\pi^{\prime}}\right)$ [11, Chap. 3, Prop. 3.4.3]. En particulier $\pi^{\prime}$ est un carré dans $F$.

Le discriminant du polynôme $X^{e}-\pi^{\prime}$ étant

$$
(-1)^{e(e-1) / 2} e^{e}\left(-\pi^{\prime}\right)^{e-1}=(-1)^{e / 2+1} e^{e} \pi^{\prime e-1},
$$

on voit que $\delta_{F / E}=(-1)^{e / 2+1} \pi^{\prime} \bmod E^{\cdot 2}$. Il en résulte que

$$
\begin{aligned}
\left(\frac{u_{\pi}}{\mathfrak{p}_{E}}\right) & =\left(\frac{\delta_{F / E} \pi^{-1}}{\mathfrak{p}_{E}}\right)=\left(\frac{(-1)^{e / 2+1} \pi^{\prime} \pi^{-1}}{\mathfrak{p}_{E}}\right) \\
& =\left(\frac{-1}{\mathfrak{p}_{E}}\right)^{e / 2+1}\left(\frac{\pi^{\prime} \pi^{-1}}{\mathfrak{p}_{E}}\right)=\left(\frac{-1}{q_{E}}\right)^{e / 2+1}\left(\frac{\pi^{\prime} \pi^{-1}}{\mathfrak{p}_{E}}\right) .
\end{aligned}
$$


Comme $F / E$ est totalement ramifiée, les deux extensions non-ramifiées $E\left(\sqrt{\pi^{\prime} \pi^{-1}}\right) / E$ et $F\left(\sqrt{\pi^{\prime} \pi^{-1}}\right) / F$ sont de même degré et nous avons

$$
\left(\frac{\pi^{\prime} \pi^{-1}}{\mathfrak{p}_{E}}\right)=\left(\frac{\pi^{\prime} \pi^{-1}}{\mathfrak{p}_{F}}\right) \text {. }
$$

Or $\pi^{\prime}$ est un carré de $F$, donc

$$
\left(\frac{\pi^{\prime} \pi^{-1}}{\mathfrak{p}_{E}}\right)=\left(\frac{\pi^{-1}}{\mathfrak{p}_{F}}\right)=\left(\frac{\pi}{\mathfrak{p}_{F}}\right)
$$

d'où le lemme.

Lorsque l'extension locale $F / E$ n'est pas totalement ramifiée, par un dévissage on peut généraliser le lemme précédent de la façon suivante :

LEMME 3.2. On suppose que l'extension locale $F / E$ est modérément ramifiée d'indice de ramification pair e et de degré résiduel $f$. Pour toute uniformisante $\pi$ de $E$, il existe une unité $u_{\pi}$ de $E$ telle que

(i) $\delta_{F / E}=\pi^{f} u_{\pi} \bmod E^{\cdot 2}$;

(ii) $\left(\frac{u_{\pi}}{\mathfrak{p}_{E}}\right)=\left(\frac{-1}{q_{E}}\right)^{f(e / 2+1)}\left(\frac{\pi}{\mathfrak{p}_{F}}\right)$.

Démonstration. La démonstration imite celle du lemme 4 de [1]. Soit $E^{\prime}$ le corps d'inertie de l'extension $F / E$. Par la formule de transitivité des discriminants [9, Chap. 3, Prop. 8], nous avons

$$
\delta_{F / E}=\delta_{E^{\prime} / E}^{e} N_{E^{\prime} / E}\left(\delta_{F / E^{\prime}}\right) \bmod E^{\cdot 2}=N_{E^{\prime} / E}\left(\delta_{F / E^{\prime}}\right) \bmod E^{\cdot 2} .
$$

L'extension $E^{\prime} / E$ étant non-ramifiée, $\pi$ reste une uniformisante de $E^{\prime}$, donc d'après le lemme précédent il existe une unité $u_{\pi}^{\prime}$ de $E^{\prime}$ telle que

$$
\delta_{F / E^{\prime}}=\pi u_{\pi}^{\prime} \bmod E^{\prime 2}
$$

et

$$
\left(\frac{u_{\pi}^{\prime}}{\mathfrak{p}_{E^{\prime}}}\right)=\left(\frac{-1}{q_{E^{\prime}}}\right)^{e / 2+1}\left(\frac{\pi}{\mathfrak{p}_{F}}\right)=\left(\frac{-1}{q_{E}}\right)^{f(e / 2+1)}\left(\frac{\pi}{\mathfrak{p}_{F}}\right) .
$$

Par ailleurs, $\left(\frac{u_{\pi}^{\prime}}{\mathfrak{p}_{E^{\prime}}}\right)$ peut être vu comme le symbole de Hilbert $\left(\frac{\pi, u_{\pi}^{\prime}}{\mathfrak{p}_{E^{\prime}}}\right)[8$, Chap. III, §5], d'où

$$
\begin{aligned}
\left(\frac{u_{\pi}^{\prime}}{\mathfrak{p}_{E^{\prime}}}\right) & =\left(u_{\pi}^{\prime}, E^{\prime}(\sqrt{\pi}) / E^{\prime}\right)(\sqrt{\pi}) / \sqrt{\pi} \\
& =\left(N_{E^{\prime} / E}\left(u_{\pi}^{\prime}\right), E(\sqrt{\pi}) / E\right)(\sqrt{\pi}) / \sqrt{\pi} \\
& =\left(\frac{\pi, N_{E^{\prime} / E}\left(u_{\pi}^{\prime}\right)}{\mathfrak{p}_{E}}\right)=\left(\frac{N_{E^{\prime} / E}\left(u_{\pi}^{\prime}\right)}{\mathfrak{p}_{E}}\right) .
\end{aligned}
$$


Maintenant si on pose $u_{\pi}=N_{E^{\prime} / E}\left(u_{\pi}^{\prime}\right)$, on obtient à la fois les deux propriétés (i) et (ii) de l'énoncé.

Le cas où l'indice de ramification $e$ de l'extension locale $F / E$ est impair a été traité dans [1] :

Lemme 3.3. On suppose que l'indice de ramification e de F/E est impair. Soit $f$ le degré résiduel de $F / E$. Alors nous avons

$$
\left(\frac{\delta_{F / E}}{\mathfrak{p}_{E}}\right)=(-1)^{f+1}\left(\frac{q_{E}}{e}\right)^{f}
$$

Démonstration. C'est le lemme 4 de [1].

\section{Globalisation}

Démonstration de la proposition 2.1. Soient $\pi$ et $\pi^{\prime}$ deux uniformisantes de $K_{\mathfrak{p}}$. Posons $u=\pi / \pi^{\prime}$.

Soit $\mathfrak{P}_{i}$ une des places de $L$ au-dessus de $\mathfrak{p}$. Désignons par $K_{\mathfrak{p}}$ et $L_{\mathfrak{P}_{i}}$ les complétés de $K$ et $L$ en les places $\mathfrak{p}$ et $\mathfrak{P}_{i}$ respectivement.

L'idéal premier $\mathfrak{p}$ étant supposé non 2-adique, l'extension $K_{\mathfrak{p}}(\sqrt{u}) / K_{\mathfrak{p}}$ est non-ramifiée. On en déduit aussitôt que si le degré résiduel correspondant $f_{i}$ est pair, alors $K_{\mathfrak{p}}(\sqrt{u})$ est contenu dans $L_{\mathfrak{P}_{i}}$ de sorte que $\left(\frac{u}{\mathfrak{P}_{i}}\right)=1$; et que si au contraire $f_{i}$ est impair, alors $\left[K_{\mathfrak{p}}(\sqrt{u}): K_{\mathfrak{p}}\right]=\left[L_{\mathfrak{P}_{i}}(\sqrt{u}): L_{\mathfrak{P}_{i}}\right]$ de sorte que $\left(\frac{u}{\mathfrak{p}}\right)=\left(\frac{u}{\mathfrak{P}_{i}}\right)$.

On ne s'intéresse dans cette proposition qu'aux idéaux premiers $\mathfrak{P}_{i}$ avec $e_{i}$ pair. Pour un tel idéal premier $\mathfrak{P}_{i}$, le lemme d'Abhyankar [7, Chap. 5, $\S 2$, Cor. 4 au Th. 5.11] garantit la non-nullité de $\left(\frac{\pi}{\mathfrak{P}_{i}}\right)$ puisqu'il affirme que l'extension $K_{\mathfrak{P}_{i}}(\sqrt{\pi}) / K_{\mathfrak{P}_{i}}$ est non-ramifiée.

Supposons maintenant qu'il y a un nombre pair d'idéaux $\mathfrak{P}_{i}$ avec $e_{i}$ pair et $f_{i}$ impair. Alors d'après ce qui précède

$$
\prod_{2 \mid e_{i}}\left(\frac{u}{\mathfrak{P}_{i}}\right)=\prod_{\substack{2 \nmid f_{i} \\ 2 \mid e_{i}}}\left(\frac{u}{\mathfrak{P}_{i}}\right)=\prod_{\substack{2 \nmid f_{i} \\ 2 \mid e_{i}}}\left(\frac{u}{\mathfrak{p}}\right)=1
$$

de sorte que

$$
\prod_{2 \mid e_{i}}\left(\frac{\pi}{\mathfrak{P}_{i}}\right)=\prod_{2 \mid e_{i}}\left(\frac{\pi^{\prime}}{\mathfrak{P}_{i}}\right) .
$$

La proposition est donc démontrée.

Démonstration de la proposition 2.4. Fixons-nous provisoirement un idéal premier $\mathcal{P}$ de $M$ au-dessus de $\mathfrak{p}$. Notons $e=e(\mathcal{P} / \mathfrak{p})$ l'indice de ramification de $\mathcal{P}$ dans l'extension $M / K$. Choisissons une uniformisante $\pi$ du 
corps local $K_{\mathfrak{p}}$ appartenant à $K$. Nous allons évaluer le produit de symboles d'Artin

$$
\prod_{\substack{\mathfrak{P}|\mathcal{P} \\ 2| e(\mathfrak{P} / \mathcal{P})}}(\mathfrak{P}, L(\sqrt{\pi}) / L)
$$

suivant la parité de $e$.

Si $e$ est pair, alors $M(\sqrt{\pi}) / M$ est non-ramifiée en $\mathcal{P}$, et nous avons

$$
\begin{aligned}
\prod_{\substack{\mathfrak{P}|\mathcal{P} \\
2| e(\mathfrak{P} / \mathcal{P})}}(\mathfrak{P}, L(\sqrt{\pi}) / L) & =\prod_{\substack{\mathfrak{P}|\mathcal{P} \\
2| e(\mathfrak{P} / \mathcal{P})}}\left(\mathcal{P}^{f(\mathfrak{P} / \mathcal{P})}, M(\sqrt{\pi}) / M\right) \\
& =(\mathcal{P}, M(\sqrt{\pi}) / M)^{\sum_{\mathfrak{P}|\mathcal{P}, 2| e(\mathfrak{P} / \mathcal{P})} f(\mathfrak{P} / \mathcal{P}) .}
\end{aligned}
$$

Comme par hypothèse $\varepsilon_{L / M}(\mathcal{P})$ est non-nul, la somme en exposant est paire de sorte que

$$
\prod_{\mathfrak{P}|\mathcal{P}, 2| e(\mathfrak{P} / \mathcal{P})}(\mathfrak{P}, L(\sqrt{\pi}) / L)=1 .
$$

Supposons maintenant que l'indice de ramification $e$ est impair. Soit $w$ une uniformisante de $M_{\mathcal{P}}$. Il existe une unité de $M_{\mathcal{P}}$ telle que $\pi=$ $w^{e} u$. Puisque $\sum_{\mathfrak{P}|\mathcal{P}, 2| e(\mathfrak{P} / \mathcal{P})} f(\mathfrak{P} / \mathcal{P})$ est pair, nous voyons, comme dans la démonstration de la proposition précédente (prop. 2.1), que

$$
\prod_{\substack{\mathfrak{P}|\mathcal{P} \\ 2| e(\mathfrak{P} / \mathcal{P})}}\left(\frac{u}{\mathfrak{P}}\right)=1
$$

de sorte que

$$
\prod_{\substack{\mathfrak{P}|\mathcal{P} \\ 2| e(\mathfrak{P} / \mathcal{P})}}(\mathfrak{P}, L(\sqrt{\pi}) / L)=\prod_{\substack{\mathfrak{P}|\mathcal{P} \\ 2| e(\mathfrak{P} / \mathcal{P})}}\left(\frac{\pi}{\mathfrak{P}}\right)=\prod_{\substack{\mathfrak{P}|\mathcal{P} \\ 2| e(\mathfrak{P} / \mathcal{P})}}\left(\frac{w}{\mathfrak{P}}\right)=\varepsilon_{L / M}(\mathcal{P}) .
$$

Ainsi lorsque $\mathcal{P}$ parcourt les idéaux premiers de $M$ au-dessus de $\mathfrak{p}$, on a

$$
\prod_{\mathcal{P} \mid \mathfrak{p}} \prod_{\substack{\mathfrak{P}|\mathcal{P} \\ 2| e(\mathfrak{P} / \mathcal{P})}}(\mathfrak{P}, L(\sqrt{\pi}) / L)=\prod_{\substack{\mathcal{P} \mid \mathfrak{p} \\ 2 \nmid e(\mathcal{P} / \mathfrak{p})}} \varepsilon_{L / M}(\mathcal{P}) .
$$

Pour obtenir la formule de la proposition, il nous faut également calculer le produit

$$
\prod_{\substack{\mathfrak{P} \mid \mathcal{P} \\ 2 \nmid e(\mathfrak{P} / \mathcal{P})}}(\mathfrak{P}, L(\sqrt{\pi}) / L)
$$

pour chaque idéal premier $\mathcal{P}$ de $M$ tel que l'indice de ramification $e=e(\mathcal{P} / \mathfrak{p})$ est pair. Comme précédemment, puisque $M(\sqrt{\pi}) / M$ est non-ramifié en $\mathcal{P}$, nous avons 


$$
\begin{aligned}
\prod_{\substack{\mathfrak{P} \mid \mathcal{P} \\
2 \nmid e(\mathfrak{P} / \mathcal{P})}}(\mathfrak{P}, L(\sqrt{\pi}) / L) & =\prod_{\substack{\mathfrak{P} \mid \mathcal{P} \\
2 \nmid e(\mathfrak{P} / \mathcal{P})}}\left(\mathcal{P}^{f(\mathfrak{P} / \mathcal{P})}, M(\sqrt{\pi}) / M\right) \\
= & (\mathcal{P}, M(\sqrt{\pi}) / M)^{\sum_{\mathfrak{P} \mid \mathcal{P}, 2 \nmid e(\mathfrak{P} / \mathcal{P})} f(\mathfrak{P} / \mathcal{P})} \\
= & (\mathcal{P}, M(\sqrt{\pi}) / M)^{\sum_{\mathfrak{P} \mid \mathcal{P}} e(\mathfrak{P} / \mathcal{P}) f(\mathfrak{P} / \mathcal{P})} \\
& =(\mathcal{P}, M(\sqrt{\pi}) / M)^{[L: M]}
\end{aligned}
$$

et ensuite

$$
\prod_{\substack{\mathcal{P}|\mathfrak{p} \\ 2| e(\mathcal{P} / \mathfrak{p})}} \prod_{\substack{\mathfrak{P} \mid \mathcal{P} \\ 2 \nmid e(\mathfrak{P} / \mathcal{P})}}(\mathfrak{P}, L(\sqrt{\pi}) / L)=\varepsilon_{M / K}(\mathfrak{p})^{[L: M]} .
$$

La formule de la proposition se déduit alors sans difficulté des considérations précédentes.

Tous les ingrédients sont maintenant réunis pour obtenir le théorème principal. Nous utiliserons les résultats locaux établis dans la section précédente, en remplaçant $E$ et $F$ par les corps locaux $K_{\mathfrak{p}}$ et $L_{\mathfrak{P}_{i}}$ respectivement.

Démonstration du théorème 2.5. L'idéal premier $\mathfrak{p}$ étant modérément ramifié dans $L$, la valuation $\mathfrak{p}$-adique du discriminant $\delta_{L / K}$ satisfait à la congruence

$$
v_{\mathfrak{p}}\left(\delta_{L / K}\right) \equiv \sum_{i=1}^{g}\left(e_{i}-1\right) f_{i} \bmod 2 \equiv \sum_{2 \mid e_{i}} f_{i} \bmod 2 .
$$

Donc si $\sum_{2 \mid e_{i}} f_{i}$ est impair, alors le symbole $\left(\frac{\delta_{L / K}}{\mathfrak{p}}\right)$ est nul comme l'est $\varepsilon_{L / K}(\mathfrak{p})$.

Plaçons-nous désormais dans la situation où $\sum_{2 \mid e_{i}} f_{i}$ est pair : Pour tout $i=1, \ldots, g$, notons $\delta_{i}=\delta_{L_{\mathfrak{P}_{i}} / K_{\mathfrak{p}}}$. Fixons-nous une uniformisante $\pi$ de $K_{\mathfrak{p}}$. Pour chaque $i$ tel que $e_{i}$ est pair, notons $u_{i}$ l'unité $u_{\pi}$ de $K_{\mathfrak{p}}$ intervenant dans le lemme 3.2. Alors, modulo les carrés de $K_{\mathfrak{p}}$, nous avons

$$
\delta_{L / K}=\prod_{i=1}^{g} \delta_{i}=\prod_{2 \nmid e_{i}} \delta_{i} \prod_{2 \mid e_{i}} \delta_{i}=\prod_{2 \nmid e_{i}} \delta_{i} \prod_{2 \mid e_{i}} u_{i}
$$

de sorte que

$$
\begin{aligned}
\left(\frac{\delta_{L / K}}{\mathfrak{p}}\right) & =\prod_{2 \nmid e_{i}}(-1)^{1+f_{i}}\left(\frac{q}{e_{i}}\right)^{f_{i}} \prod_{2 \mid e_{i}}\left(\frac{-1}{q}\right)^{f_{i}\left(e_{i} / 2+1\right)}\left(\frac{\pi}{\mathfrak{P}_{i}}\right) \\
& =(-1)^{F}\left(\frac{q}{E}\right)(-1)^{((q-1) / 2) \sum_{2 \mid e_{i}} f_{i}\left(e_{i} / 2+1\right)} \varepsilon_{L / K}(\mathfrak{p}) \\
& =(-1)^{F+(q-1) G / 2}\left(\frac{q}{E}\right) \varepsilon_{L / K}(\mathfrak{p}) .
\end{aligned}
$$


Démonstration de la proposition 2.8. Soient $K_{\mathfrak{p}}$ et $L_{\mathfrak{P}}$ les complétés de $K$ et $L$ en les places $\mathfrak{p}$ et $\mathfrak{P}$ respectivement. Notons $E$ le sous-corps de $L_{\mathfrak{P}}$ laissé fixe par le 2-sous-groupe de Sylow de $G\left(L_{\mathfrak{P}} / K_{\mathfrak{p}}\right) \simeq D_{\mathfrak{P}}(L / K)$. Comme $f$ est supposé pair, l'extension quadratique non-ramifiée $M$ de $E$ est contenue dans $L_{\mathfrak{P}}$. Puisque $\left[E: K_{\mathfrak{p}}\right.$ ] est un entier impair, l'extension $E(\sqrt{\pi}) / E$ est non-triviale et ramifiée de sorte que l'extension $M(\sqrt{\pi}) / E$ est galoisienne de groupe de Galois $\mathbb{Z} / 2 \mathbb{Z} \times \mathbb{Z} / 2 \mathbb{Z}$.

Ceci étant, si $\left(\frac{\pi}{\mathfrak{P}}\right)=1$, alors $M(\sqrt{\pi}) \subset L_{\mathfrak{P}}$ et le groupe de Galois $G\left(L_{\mathfrak{P}} / E\right)$ se surjecte dans $\mathbb{Z} / 2 \mathbb{Z} \times \mathbb{Z} / 2 \mathbb{Z}$. Il n'est donc pas cyclique. Réciproquement, si le 2-groupe $G\left(L_{\mathfrak{P}} / E\right)$ n'est pas cyclique, alors il existe un corps $F$ entre $E$ et $L_{\mathfrak{P}}$ qui possède deux extensions quadratiques distinctes contenues dans $L_{\mathfrak{P}}$. Comme le corps local $F$ n'est pas 2-adique, cela entraîne que toutes les extensions quadratiques de $F$ sont contenues dans $L_{\mathfrak{P}}$. En particulier, l'uniformisante $\pi \in K_{\mathfrak{p}} \subseteq F$ est un carré dans $L_{\mathfrak{P}}$, autrement $\operatorname{dit}\left(\frac{\pi}{\mathfrak{P}}\right)=1$.

Nous remarquons que la parité du degré résiduel $f$ n'est en fait utilisée que dans un sens de l'équivalence de la proposition précédente. En effet, lorsque le 2-sous-groupe de Sylow de $G\left(L_{\mathfrak{P}} / K_{\mathfrak{p}}\right)$ n'est pas cyclique alors, comme on vient de voir au cours de la démonstration précédente, $\left(\frac{\pi}{\mathfrak{P}}\right)=1$ sans aucune hypothèse sur $f$.

5. Exemples. Nous terminons avec deux familles d'exemples. Rappelons qu'on est toujours dans le cas où la ramification est modérée.

1. Plaçons-nous dans la situation où il existe un corps intermédiaire $M$ entre $K$ et $L$, et où l'idéal premier $\mathfrak{p}$ de $K$ ne se décompose pas dans $M$. Notons $\mathcal{P}$ l'idéal premier de $M$ au-dessus de $\mathfrak{p}$, alors $\mathfrak{p}=\mathcal{P}^{e(\mathcal{P} / \mathfrak{p})}$.

Supposons que l'indice de ramification $e=e(\mathcal{P} / \mathfrak{p})$ est pair tandis que le degré résiduel $f=f(\mathcal{P} / \mathfrak{p})$ est impair; autrement dit $\varepsilon_{M / K}(\mathfrak{p})=0$. Soient $\mathfrak{P}_{1}, \ldots, \mathfrak{P}_{g}$ les idéaux premiers de $L$ au-dessus de $\mathfrak{p}$ et $f_{1}, \ldots, f_{g}$ leurs degrés résiduels respectifs.

Si $\sum_{i=1}^{g} f_{i}$ est impair, alors $\varepsilon_{L / K}(\mathfrak{p})=\left(\frac{\delta_{L / K}}{\mathfrak{p}}\right)=0$. Si, au contraire, $\sum_{i=1}^{g} f_{i}$ est pair, alors

$$
\varepsilon_{L / K}(\mathfrak{p})=1 \quad \text { et } \quad\left(\frac{\delta_{L / K}}{\mathfrak{p}}\right)=(-1)^{(q-1) G / 2} \quad \text { où } G=\sum_{\substack{4 \mid e_{i} \\ 2 \nmid f_{i}}} 1 .
$$

En effet, soit $\pi \in \mathfrak{p}$ une uniformisante de $K_{\mathfrak{p}}$. Par le lemme d'Abhyankar, l'extension $M(\sqrt{\pi}) / M$ est non-ramifiée en $\mathcal{P}$ de sorte que le symbole d'Artin $(\mathcal{P}, M(\sqrt{\pi}) / M)$ est bien défini. Comme $f$ est supposé impair, nous avons également $\sum_{i=1}^{g} f\left(\mathfrak{P}_{i} / \mathcal{P}\right)$ est pair. D'où

$$
(\mathcal{P}, M(\sqrt{\pi}) / M)^{\sum_{i=1}^{g} f\left(\mathfrak{P}_{i} / \mathcal{P}\right)}=\mathrm{Id} .
$$


On en déduit, par la fonctorialité du symbole d'Artin, que la restriction à $M(\sqrt{\pi})$ de $\left(\prod_{i=1}^{g} \mathfrak{P}_{i}, L(\sqrt{\pi}) / L\right)$ est l'identité. Donc

$$
\left(\prod_{i=1}^{g} \mathfrak{P}_{i}, L(\sqrt{\pi}) / L\right)=\mathrm{Id},
$$

ce qui signifie bien que $\varepsilon_{L / K}(\mathfrak{p})=1$. La formule $\left(\frac{\delta_{L / K}}{\mathfrak{p}}\right)=(-1)^{(q-1) G / 2}$ en est alors une conséquence immédiate.

2. Plaçons-nous dans la situation où il existe une extension cyclique $M$ de $K$ contenue dans $L$, et où l'idéal premier $\mathfrak{p}$ de $K$ ne se décompose pas dans $M$. Notons $\mathcal{P}$ l'idéal premier de $M$ au-dessus de $\mathfrak{p}$, alors $\mathfrak{p}=\mathcal{P}^{e(\mathcal{P} / \mathfrak{p})}$.

Supposons que l'indice de ramification $e=e(\mathcal{P} / \mathfrak{p})$ et le degré résiduel $f=f(\mathcal{P} / \mathfrak{p})$ sont pairs. Soient $\mathfrak{P}_{1}, \ldots, \mathfrak{P}_{g}$ les idéaux premiers de $L$ au-dessus de $\mathfrak{p}$ et $f_{1}, \ldots, f_{g}$ leurs degrés résiduels respectifs. Si maintenant la somme des degrés résiduels $\sum_{i=1}^{g} f\left(\mathfrak{P}_{i} / \mathcal{P}\right)$ est impaire, alors

$$
\varepsilon_{L / K}(\mathfrak{p})=\left(\frac{\delta_{L / K}}{\mathfrak{p}}\right)=-1 .
$$

En effet, soit $\pi \in \mathfrak{p}$ une uniformisante de $K_{\mathfrak{p}}$. D'après la proposition 2.8, nous avons $\varepsilon_{M / K}(\mathfrak{p})=\left(\frac{\pi}{\mathcal{P}}\right)=-1$; autrement dit l'image de $\mathcal{P}$ par le symbole d'Artin $(\quad, M(\sqrt{\pi}) / M)$ n'est pas l'identité : $(\mathcal{P}, M(\sqrt{\pi}) / M) \neq$ Id . Comme $\sum_{i=1}^{g} f\left(\mathfrak{P}_{i} / \mathcal{P}\right)$ est impair, nous avons également

$$
(\mathcal{P}, M(\sqrt{\pi}) / M)^{\sum_{i=1}^{g} f\left(\mathfrak{P}_{i} / \mathcal{P}\right)} \neq \mathrm{Id} .
$$

Toujours par la fonctorialité du symbole d'Artin, ceci entraîne

$$
\operatorname{Res}_{M(\sqrt{\pi})}\left(\prod_{i=1}^{g} \mathfrak{P}_{i}, L(\sqrt{\pi}) / L\right) \neq \mathrm{Id} .
$$

D'où évidemment $\left(\prod_{i=1}^{g} \mathfrak{P}_{i}, L(\sqrt{\pi}) / L\right) \neq \mathrm{Id}$, ce qui signifie bien que $\varepsilon_{L / K}(\mathfrak{p})$ $=-1$. L'égalité $\left(\frac{\delta_{L / K}}{\mathfrak{p}}\right)=-1$ en est alors une conséquence immédiate.

Remerciement. Les auteurs tiennent à remercier le professeur Francisco Diaz y Diaz qui leur a fourni des exemples de calcul de $\varepsilon_{L / K}(\mathfrak{p})$.

\section{Bibliographie}

[1] P. Barrucand et F. Laubie, Sur les symboles des restes quadratiques des discriminants, Acta Arith. 48 (1987), 81-88.

[2] J. P. Buhler, Icosahedral Galois Representations, Lecture Notes in Math. 654, Springer, 1978.

[3] L. E. Dickson, History of the Theory of Numbers, Vol. I, reprinted by Chelsea, 1952.

[4] D. M. Dribin, Permutation groups, Ann. of Math. 38 (1937), 739-749. 
[5] H. Hasse, Arithmetische Theorie der kubischen Zahlkörper auf klassenkörpertheoretischen Grundlage, Math. Z. 31 (1930), 565-582.

[6] G. Kientega, Sur les corps algébriques du quatrième degré, thèse de troisième cycle, Publ. Univ. Paris VI, 1980.

[7] W. Narkiewicz, Elementary and Analytic Theory of Algebraic Numbers, 2nd ed., Springer, Berlin; PWN-Polish Sci. Publ., Warszawa, 1990.

[8] J. Neukirch, Class Field Theory, Grundlehren Math. Wiss. 280, Springer, 1986.

[9] J.-P. Serre, Corps locaux, troisième édition, Hermann, Paris, 1968.

[10] G. E. Wahlin, The factorisation of the rational primes in a cubic domain, Amer. J. Math. 44 (1922), 191-203.

[11] E. Weiss, Algebraic Number Theory, reprinted by Chelsea, 1963.

\section{LACO (UPRESA 6090 CNRS)}

Département de mathématiques

Université de Limoges

123 Avenue Albert Thomas

87060 Limoges Cedex, France

E-mail: mova@unilim.fr zahidi@unilim.fr 